\title{
Effectiveness of Early Ureteric Stenting for Urosepsis Associated with Urinary Tract Calculi
}

\author{
Sho Nishiguchi ${ }^{1}$, Joel Branch ${ }^{1}$, Yu Suganami ${ }^{1}$, Izumi Kitagawa ${ }^{1}$ and Yasuharu Tokuda ${ }^{2}$
}

\begin{abstract}
Objective Patients with urosepsis associated with urinary tract calculi occasionally require drainage, primarily via ureteric stenting. Such patients require longer hospitalization. However, the indications for early ureteric stenting for this condition have not been clearly defined. To compare the length of stay (LOS) in the hospital between patients treated with earlier ureteric stenting versus those with delayed ureteric stenting.

Methods Design: Retrospective cohort study. Setting: An acute care teaching hospital in Japan. Measurement: Length of hospital stay in days.

Patients Patients with urosepsis associated with urinary tract calculi.

Results Among a total of 30 patients (mean age, 72; 13 men), the mean number of days from emergency room admission to ureteric stenting was 3.5 days (range, 1-14 days), and the overall mean LOS was 36 days (range, 8-102 days). The early stenting group (mean LOS, 21 days) had a significantly shorter LOS than the delayed stenting group (mean LOS, 50 days), with an adjusted beta coefficient of -26 days [95\% confidence interval (CI), -46, -6].

Conclusion In patients with urosepsis associated with urinary tract calculi, performing early stenting within two days of admission may reduce the LOS in the hospital.
\end{abstract}

Key words: ureteric stenting, urosepsis, urinary tract calculi, length of stay, earlier ureteric stenting, complicated acute pyelonephritis

(Intern Med 53: 2205-2210, 2014)

(DOI: 10.2169/internalmedicine.53.2617)

\section{Introduction}

Sepsis is defined as systemic inflammatory response syndrome (SIRS) related to infection. SIRS can be caused by local or systemic infections, resulting in a host of inflammatory cascades that potentially lead to multi-organ failure. A diagnosis of SIRS requires two or more of the following criteria: 1) a body temperature greater than $38^{\circ} \mathrm{C}$ or less than $36^{\circ} \mathrm{C}, 2$ ) an increased heart rate greater than 90 beats per minute, 3) tachypnea manifested as a respiratory rate greater than 20 breaths per minute or hyperventilation, as indicated by a partial carbon dioxide pressure of less than $32 \mathrm{mmHg}$ and 4) an altered white blood cell (WBC) count greater than $12,000 / \mathrm{mm}^{3}$ or less than $4,000 / \mathrm{mm}^{3}$ or more than $10 \% \mathrm{im}$ mature polymorphonuclear leukocytes (1-5). Among the many types of sepsis, one of the most common is urosepsis, which is caused by urinary tract infections (UTIs). Based on the definition of sepsis, any patient with a UTI meeting two or more of the SIRS criteria is considered to have urosepsis.

The mortality and morbidity among patients with sepsis is considerably increased when severe sepsis or septic shock ensue, although the prognosis of urosepsis is globally better than that of sepsis from other infectious causes (6). Patients with serious morbidities require a greater length of hospital stay than those without such conditions. Therefore, the treatment guidelines recommend that patients with sepsis be managed before they develop severe sepsis or septic shock using early goal-directed therapy consisting of intravenous fluids, antimicrobial agents, vasopressors and blood products (2-8).

Urosepsis is broadly categorized into uncomplicated and

${ }^{1}$ Department of General Internal Medicine, Shonan Kamakura General Hospital, Japan and ${ }^{2}$ Department of General Medicine, Mito Kyodo General Hospital, Japan

Received for publication February 4, 2014; Accepted for publication March 18, 2014

Correspondence to Dr. Sho Nishiguchi, sanazen@hotmail.co.jp 
complicated types (9). Complicated urosepsis is defined as urosepsis associated with a structural or functional abnormality of the urinary tract, including ureteric obstruction caused by urinary tract calculi. Patients with complicated urosepsis associated with urinary tract calculi occasionally require ureteric stent placement, or rarely, percutaneous nephrostomy insertion to restore and drain the infected urinary tract (8). However, the indications for ureteric stent placement have not been clearly defined, and many patients with urosepsis associated with urinary tract calculi receive stent insertion based on the individual physician's preference (10).

There have been no studies that have evaluated the appropriate timing of stent placement in such patients. Earlier placement may lead to a lower incidence of serious morbidity, and thus a shorter length of stay in the hospital, although this issue has not yet been evaluated in the literature. Therefore, in this current investigation of a retrospective cohort of patients who required stenting after admission, we aimed to assess the timing of stent placement in patients with urosepsis associated with urinary tract calculi. Specifically, we compared the length of stay (LOS) in the hospital between those treated with and without earlier stenting.

\section{Materials and Methods}

\section{Patients and setting}

A retrospective study was conducted of patients who were admitted and underwent ureteric stent placement for urosepsis associated with urinary tract calculi at our hospital between July 2008 and July 2012 (inclusive). The diagnosis of a urinary tract infection was based on the symptoms, a physical examination, ultrasound or radiological features and laboratory data, including pyuria (10 or more leukocytes per high power field in a centrifuged specimen) and urine cultures growing the same organisms as blood cultures, if such cultures were also positive. The presence of urinary tract calculi was evaluated using imaging studies (ultrasonography or computed tomography).

Urinary tract stent insertion was performed by Japanese board-certified urologists. The indication for insertion was based on the presence of multiple organ failure (elevated liver enzyme levels, an elevated serum creatinine level, a low platelet count and a prolonged coagulation time) and persistent inflammation, such as a high fever (axillary temperature $>38.0^{\circ} \mathrm{C}$ ), a high WBC count and an elevated Creactive protein level. After undergoing stent placement, the patients were further treated by physicians (residents and staff) in our Department of General Internal Medicine.

We retrospectively analyzed the clinical data of consecutive urosepsis patients treated with stenting for urinary tract calculi $(n=30)$ and compared the data between the earlier stenting group and the delayed stenting group. The earlier stenting group (15 patients) included patients treated with stent placement within two days after emergency room (ER) admission. The delayed stenting group (15 patients) included patients treated with stent placement more than two days after ER admission.

\section{Data collection}

Data were collected for each patient, including the age, sex, pain, comorbidities, days from ER admission to ureteric stent placement, LOS, vital signs (systolic blood pressure (sBP), diastolic blood pressure (dBP), mean blood pressure $(\mathrm{mBP})$, heart rate $(\mathrm{HR})$, body temperature $(\mathrm{BT})$, respiratory rate), Glasgow Coma Scale, laboratory data (WBC, platelets, total bilirubin, serum creatinine, prothrombin time, activated partial thromboplastin time, fibrin/fibrinogen degradation products, d-dimer) and calculus characteristics (size and location). The calculus size was assessed using the magnitude of the surface area calculated according to the European Association of Urology guidelines.

Organ failure was evaluated using the Sequential Organ Failure Assessment (SOFA) score (11). The SOFA score assesses the functions of six different organ systems, including: respiratory (partial arterial oxygen pressure/fraction of inspired oxygen), cardiovascular ( $\mathrm{mBP}$ and use of vasoactive drugs), renal (creatinine), hepatic (total bilirubin), neurological (Glasgow Coma Score) and hematological (platelet count) systems. The data available during the first 24 hours of admission for each patient were used to determine the SOFA score.

\section{Statistical analysis}

We aimed to evaluate our primary hypothesis that performing earlier stenting in patients with urosepsis associated with urinary tract calculi can reduce the LOS in the hospital. Since previous studies have shown that the mean period from ER admission to ureteric stent placement was approximately three days, the patients were classified into either the earlier stenting group (stent placement performed within two days after ER admission) or a delayed stenting group (stent placement performed more than two days after ER admission). The primary outcome measure was the LOS. The baseline characteristics of the two groups were compared using Fisher's exact test and the Mann-Whitney U test, where appropriate (Table 1). To evaluate the association between the timing of stent placement and the LOS, a simple linear regression model was constructed, and a multivariable adjusted linear regression model was developed using the age, sex, SOFA score and stent group (Table 2). All data analyses were carried out using the SPSS Statistics version J20 (IBM, Tokyo, Japan) and STATA version 11 (Light Stone, Texas, USA) software programs. The study was approved by the hospital ethics committee of Shonan Kamakura General Hospital.

\section{Results}

A total of 32 patients with urosepsis associated with urinary tract calculi underwent ureteric stent placement during 
Table 3. The Results of the Multivariable-adjusted Linear Regression Model for the LOS, Stratified by Gender

\begin{tabular}{|c|c|c|c|}
\hline \multicolumn{4}{|l|}{ Female } \\
\hline Variable & Beta & $95 \%$ CI of Beta & $\mathrm{p}$ value \\
\hline Stent placement $<2$ days from ER admission & -35 & $(-46$ to -25$)$ & $<0.01 *$ \\
\hline Age & -0.1 & $(-0.5$ to -0.2$)$ & 0.410 \\
\hline SOFA score & -2 & $(-3.8$ to -0.1$)$ & $0.05 *$ \\
\hline \multicolumn{4}{|l|}{ Male } \\
\hline Stent placement $<2$ days from ER admission & -6 & $\left(\begin{array}{lll}-68 & \text { to } & 56\end{array}\right)$ & 0.830 \\
\hline Age & 1 & $(-1.4$ to 2.8$)$ & 0.474 \\
\hline SOFA score & 2 & $(-6.2$ to 9.4$)$ & 0.658 \\
\hline
\end{tabular}

Table 4. Pathogens Identified in the Urosepsis Patients

\begin{tabular}{|c|c|c|c|c|}
\hline & Urine culture & Blood culture & Pyuria & Group \\
\hline 1 & Escherichia Coli & Escherichia Coli & 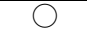 & Earlier \\
\hline 2 & Escherichia Coli & Escherichia Coli & $\bigcirc$ & Earlier \\
\hline 3 & Escherichia Coli & Escherichia Coli & 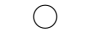 & Earlier \\
\hline 4 & Escherichia Coli & Escherichia Coli & & Earlier \\
\hline 5 & Escherichia Coli & Escherichia Coli & 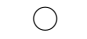 & Earlier \\
\hline 6 & Escherichia Coli & Escherichia Coli & & Earlier \\
\hline 7 & Escherichia Coli & Escherichia Coli & 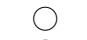 & Delayed \\
\hline 8 & Escherichia Coli & Escherichia Coli & & Delayed \\
\hline 9 & Escherichia Coli & Escherichia Coli & & Delayed \\
\hline 10 & Escherichia Coli & Escherichia Coli & & Delayed \\
\hline 11 & Escherichia Coli & Escherichia Coli & $J$ & Delayed \\
\hline 12 & Escherichia Coli & Negative & $\times$ & Delayed \\
\hline 13 & Proteus spp. & Proteus spp. & $\times$ & Earlier \\
\hline 14 & Proteus spp. & Proteus spp. & 0 & Earlier \\
\hline 15 & Proteus spp. & Proteus spp. & 0 & Earlier \\
\hline 16 & Proteus spp. & Proteus spp. & $\bigcirc$ & Delayed \\
\hline 17 & Proteus spp. & Negative & . & Earlier \\
\hline 18 & Proteus spp. & Negative & ) & Delayed \\
\hline 19 & MRSA, Pseudomonas spp., Proteus spp. & MRSA, Pseudomonas spp., Proteus spp. & & Delayed \\
\hline 20 & Klebsiella spp. & Klebsiella spp. & 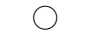 & Earlier \\
\hline 21 & Klebsiella spp. & Klebsiella spp. & $\checkmark$ & Earlier \\
\hline 22 & Klebsiella spp. & Klebsiella spp. & $\bigcirc$ & Earlier \\
\hline 23 & Negative & Klebsiella spp., CNS & $\times$ & Delayed \\
\hline 24 & Enterococcus faecalis & Enterococcus faecalis & O & Earlier \\
\hline 25 & Enterococcus faecalis & Negative & $\bigcirc$ & Delayed \\
\hline 26 & Negative & CNS & & Delayed \\
\hline 27 & Candida albicans & Streptococcus anginosus & $\bigcirc$ & Delayed \\
\hline 28 & Candida albicans & Candida albicans & & Delayed \\
\hline 29 & Staphylococcus aureus & Staphylococcus aureus & $\bigcirc$ & Earlier \\
\hline 30 & Negative & Negative & $\bigcirc$ & Delayed \\
\hline
\end{tabular}

\section{Discussion}

To the best of our knowledge, this study is the first to report the relationship between the timing of ureteric stent placement and the LOS in the hospital in patients with urosepsis associated with urinary tract calculi. Our analysis revealed that early stent intervention has the potential to reduce the hospital LOS, which was approximately 26 days less than that observed in the patients in which stenting was delayed. Because the hospital LOS of our patients was relatively long (mean, 36 days), an early stenting strategy is recommended to reduce the LOS, cost of hospitalization and potential complications associated with a longer LOS in hospitals, including catheter-associated recurrent urinary tract infections and other health care-associated infections.

There may be a difference in the willingness to accept ureteric stenting between men and women with urosepsis associated with urinary tract calculi. Ureteric stent placement in men usually requires general or spinal anesthesia because severe urethral pain is caused by the endoscopic procedure, although this is not the case in women. Therefore, many urologists do not perform ureteric stenting in men, and it is thus predictable that the delayed stenting group had a higher proportion of male subjects. However, based on the results of our sex-adjusted model, early stenting is effective in reducing the hospital LOS in men; therefore, an early stenting strategy may be recommended to reduce the LOS in both men and women. There are no clear explanations regarding the differences in the SBP and WBC count between the two 


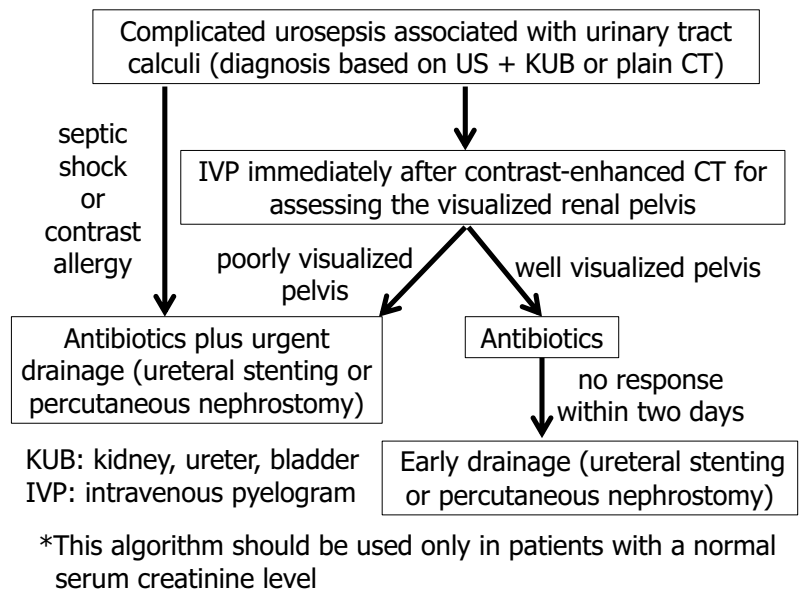

Figure. The treatment algorithm for urosepsis associated with urinary tract calculi

groups.

Three patients did not have pyuria; however, the cultures of their urine and blood revealed positive bacterial growth. One patient had no pyuria despite a positive urine culture and had been partially treated with antibiotics before arriving at our hospital. There are two possible explanations for these results; the ineffective collection of urine for the analysis and/or that the partial treatment resulted in only a few bacteria being identified in the urine, which was not sufficient to induce pyuria. Another patient had a positive urine culture with only scant bacteria that did not induce pyuria. In the remaining patient, the urinary tract was completely obstructed by urinary tract calculi, and the blood cultures were positive despite the absence of pyuria and negative urine cultures.

\section{Comparison with other studies}

All patients were discharged alive in our study. In a previous study (1), one patient died despite undergoing stenting. There may have been a difference in the indications for ureteric stenting between the study groups that accounted for this difference. Since the omission of stenting is a potential risk factor for mortality in this patient population, the use of this treatment should be analyzed as a crucial factor in further research investigating the optimal treatment for these patients.

Compared to previous studies $(1,12)$, the mean number of days from ER admission to ureteric stenting (3.5 days) and the mean LOS (36 days) were longer in our study. In our hospital, emergency physicians usually admit most patients with urosepsis to the Department of General Internal Medicine. When a diagnosis of urosepsis associated with urinary tract calculi is established, a consultation with urologists is then undertaken; thus, there may have been greater time intervals from ER admission to the decision to perform ureteric stenting because of this consultation process. When caring for complicated urosepsis patients, the urologist should be informed of such patients expeditiously to prevent any delay in performing ureteric stenting. The appropriate use of stenting in such patients, including the timing of placement, can be achieved by establishing an in-hospital protocol regarding the indications for ureteric stenting in patients with urosepsis associated with urinary tract calculi.

One observational study (13) conducted in a teaching hospital in Okinawa, Japan showed the usefulness of performing intravenous pyelography (IVP) immediately after obtaining a contrast-enhanced abdominal computed tomography (CT) scan in affected patients. When the renal pelvis in the affected kidney cannot be visualized, this is due to a delay in contrast delivery to the collecting system, and is considered to be a sign of complete ureteric obstruction, which is considered to be an indication for urgent ureteric stenting. Overall, the authors' recommendation is for urgent ureteric stenting to be performed in all patients with septic shock, or in those with a poorly visualized renal pelvis on IVP after obtaining a contrast-enhanced abdominal CT scan (13). This strategy, along with our results, may be worth considering when establishing a treatment algorithm for such patients. Figure shows our proposed treatment algorithm after assessing the extent of hydronephrosis and confirming the presence of urinary tract calculi on ultrasonography (US) + Kidney-Ureter-Bladder (KUB) or abdominal plain CT.

\section{Limitations of the study}

First, the present study was based on a single-site cohort, and the sample size was small. Therefore, studies with larger sample sizes and multiple institutions are needed. Our small sample size may have led to type 2 errors (false negative results) for other independent variables (age, gender and SOFA score) associated with the dependent variable. Second, this was a retrospective observational study. Randomized controlled trials are usually needed to determine the efficacy of strategies used for intervention, such as ureteric stenting. In order to conduct such a trial, it is appropriate to establish a state-of-the-art treatment algorithm, including the exact timing for stenting, for example within 24 vs. 48 hours after ER admission, in patients with urosepsis associated with urinary tract calculi and to perform a trial evaluating the treatment algorithm in comparison to the traditional approach in prospective patients.

\section{Areas for future research}

Determining the appropriate indications and timing for ureteric stenting in this area is of great importance; thus, additional research is needed to evaluate the guidelines and algorithms, such as ours, for the timing of ureteric stenting in this patient population. This would be helpful for improving the survival and shortening the LOS, as well as reducing the costs and complications associated with hospital stays. Prospective studies conducted by multiple institutions would be most appropriate due to the potential differences in procedural quality among urologists at different hospitals. 


\section{Conclusion}

The results of our retrospective study revealed that performing earlier stenting within two days in patients with urosepsis associated with urinary tract calculi reduces the LOS in the hospital. Shortening the LOS may reduce the costs and complications associated with a longer LOS. We herein propose a treatment algorithm based on both our results, as well as previous recommendations. Prospective studies evaluating this algorithm are therefore called for.

The authors state that they have no Conflict of Interest (COI).

\section{References}

1. Yoshimura K, Utsunomiya N, Ichioka K, Ueda N, Matsui Y, Terai A. Emergency drainage for urosepsis associated with upper urinary tract calculi. J Urol 173: 458-462, 2005.

2. Marx G, Reinhart K. Urosepsis: from the intensive care viewpoint. Int J Antimicrob Agents 31: 79-84, 2008.

3. Bone RC, Balk RA, Cerra FB, et al. Definitions for sepsis and organ failure and guidelines for the use of innovative therapies in sepsis. The ACCP/SCCM Consensus Conference Committee. American College of Chest Physicians/Society of Critical Care Medicine. Chest 101: 1644-1655, 1992.
4. American College of Chest Physicians/Society of Critical Care Medicine Consensus Conference. Definitions for sepsis and organ failure and guidelines for the use of innovative therapies in sepsis. Crit Care Med 20: 864-874, 1992.

5. Levy MM, Fink MP, Marshall JC, et al. 2001 SCCM/ESICM/ ACCP/ATS/SIS International Sepsis Definitions Conference. Intensive Care Med 29: 530-538, 2003.

6. Wagenlehner FM, Weidner W, Naber KG. Optimal management of urosepsis from the urological perspective. Int J Antimicrob Agents 30: 390-397, 2007.

7. Annane D, Bellissant E, Cavaillon JM. Septic shock. Lancet $\mathbf{3 6 5}$ : 63-78, 2005.

8. Berger I. Emergency nephrectomy due to severe urosepsis: a retrospective, multicentre analysis of 65 cases. BJU Int 104: 386-390, 2009.

9. Pallett A, Hand K. Complicated urinary tract infections: practical solutions for the treatment of multiresistant Gram-negative bacteria. J Antimicrob Chemother 65: 25-33, 2010.

10. Christoph F. How septic is urosepsis? Clinical course of infected hydronephrosis and therapeutic strategies. World J Urol 23: 243247, 2005.

11. Ulvik A. Multiple organ failure after trauma affects even longterm survival and functional status. Crit Care 11: 95, 2007.

12. Yamamoto Y, Fujita K, Nakazawa $S$, et al. Clinical characteristics and risk factors for septic shock in patients receiving emergency drainage for acute pyelonephritis with upper urinary tract calculi. BMC Urol 12: 4, 2012.

13. Arakaki Y. Emergency urological disease. Kyukyuigaku (Emergency Medicine) 34: 199-205, 2010 (in Japanese, Abstract in English).

(C) 2014 The Japanese Society of Internal Medicine http://www.naika.or.jp/imonline/index.html 